\title{
»...WHEN I'M NOT PUT ON THIS LIST...« KANONISIERUNGSPROZESSE IM HIPHOP AM BEISPIEL EMINEM
}

\section{Oliver Kautny}

Auf den ersten Blick mag Kanonisierung im HipHop als transparenter Prozess erscheinen, der sich dem Beobachter der Szene leicht erschließt. Denn die HipHop-Szene stellt ganz offen die Frage, welche ihrer Songs, Alben und Musiker so maßgeblich sind, dass sie es verdienen, zum vorbildlichen und erinnerungswürdigen Kernbestand, zum Kanon also, zu gehören. Dabei fältt etwa auf, dass es zur anerkannten Sprachpraxis gehört, sich als Rapper selbst zur Elite des HipHop zu zählen, um so den zukünftigen Klassikerstatus der eigenen Musik vorwegzunehmen. Diese als boasting bezeichnete Selbstpreisung gehört genauso wie die Schmähung der Gegner (dissing) zur genrespezifischen Wettkampfkultur, die sich nicht nur in konzertähnlichen RapBattles ausdrückt, sondern auch in Battle-Songs auf Alben, in langwierigen Fehden zwischen Rappern (beefs) sowie in als Wettkampf inszenierten, zeitgleichen CD-Veröffentlichungen mehrerer Rapper. Genährt wird dieses System des Battles von der Hoffnung der Konkurrenten, rezipiert, respektiert, verehrt und erinnert zu werden. In der Sprache der Szene sind es in erster Linie realness (Authentizität), aber auch skills (sprachliche und musikalische Fertigkeiten) und style (Originalität), die den Zugang zum RapOlymp rechtfertigen. Und je stärker im Diskurs die Kräfte des Mainstreaming zum Tragen kommen, desto stärker scheint auch fame (Klein/Friedrich 2003: 40-43), insbesondere in Gestalt kommerziellen Erfolges, als Richtschnur der Bewertung zu gelten.

Wie ich an einem Beispiel der Eminem-Rezeption in den USA aufzeigen werde, wirken unter der Oberfläche dieser im Wettstreit zu Tage tretenden Wertungsdiskurse jedoch auch soziale sowie wirtschaftliche Machtinteressen der jeweiligen Diskursteilnehmer auf den Prozess der HipHop-Kanonisierung ein. Um dieses Ineinandergreifen von ästhetischen und soziologischen Faktoren näher zu beleuchten, widme ich mich im Folgenden der Fehde zwischen 
dem US-amerikanischen HipHop-Magazin The Source und Eminem, einem regelrechten Kampf um den Mainstream-Kanon des HipHop in der Arena von Musikkritik und Musikwirtschaft. Nach der Klärung des hier verwendeten Kanonbegriffes werde ich die Sichtbarmachung, Selbstpositionierung und Rezeption Eminems innerhalb der Kanondebatten darstellen.

\section{Kanonisierungsprozesse im HipHop}

Wenn im Folgenden von Kanonisierungsprozessen die Rede sein wird, ist damit im Anschluss an Jan Assmann der Versuch einer Kommunikationsgemeinschaft gemeint, inhaltlich fixierte, normative Wertmaßstäbe zu definieren bzw. eine normprägende Sammlung (etwa von Songs oder Interpreten) auszubilden. Vor allem das Erstellen von Werk- oder Künstlerlisten ist eine Form kultureller Erinnerungsarbeit und schafft Wertorientierung und Identität in der Gegenwart (Assmann 2005: 119ff.). »Ein Kanon definiert die Maßstäbe dessen, was als schön, groß und bedeutsam zu gelten hat. Und er tut das, indem er auf Werke verweist, die solche Werte in exemplarischer Weise verkörpern« (ebd.: 119). Der Grad der Abgeschlossenheit kanonischer Listen variiert dabei beträchtlich. Zum einen spricht Assmann mit Blick auf das Altertum erst dann von einem Kanon, wenn der unberechenbare Strom der Tradition und ihr Prozess des Anthologisierens, Erinnerns, Vergessens, Hinzukommens und Streichens von Werken oder Werten gestoppt wird, wie es dem Christentum etwa mit der Bibel, dem Prototyp des Kanons, gelungen ist. Zum anderen wendet Assmann den Kanonbegriff für neuzeitliche Phänomene, in Analogie zum allgemeinen Sprachgebrauch, auch auf nicht abgeschlossene und wandelbare Werklisten und Prinzipienkataloge an, etwa auf den Kanon der klassischen Musik oder des Feminismus (ebd.: 91-94, 129). Wenn im HipHop von Kanonisierung gesprochen werden kann, dann nur im letzteren prozesshaften Sinne, denn zu einer letztgültigen normativen Wertsetzung und Listung von HipHop-Songs, -Alben sowie -Interpreten kam es bisher nicht. Insgesamt sind Kanonisierungsprozesse im HipHop übrigens als instabil zu bezeichnen, vor allem verglichen mit dauerhaften Kanones im Rock (Appen/Doehring 2006: 24). Gründe hierfür zu suchen, wäre ein gesondertes Thema. Ich vermute, dass die ausgeprägte Battle-Struktur des HipHop zur Kurzlebigkeit und Unabgeschlossenheit seiner Kanones erheblich beiträgt.

Antti-Ville Kärjä (2006: 9-17) schlägt im Anschluss an Philip V. Bohlman vor, zwischen drei Formen der Kanonformation populärer Musik zu unterscheiden: »alternative canon«, »mainstream canon« und »prescribed canon«. 
Die Unterscheidung zwischen alternativen Kanones und Mainstream-Kanones bezieht sich vorwiegend auf die sozial-ökonomische Struktur der Wertungsdiskurse und der kanonischen Listen, die sie produzieren. Kleinere Kommunikationsgemeinschaften mit weniger weit reichenden, oft lokal gebundenen Kommunikationskanälen bilden nach Kärja alternative Kanones aus, inhaltlich meist bezogen auf entlegenere Subgenres im so genannten Underground populärer Musik. Größere Kommunikationsgemeinschaften mit nationaler und transnationaler Reichweite verhandeln in der Regel musikwirtschaftlich überregional distribuierte und massenmedial vermittelte Musik und formen Mainstream-Kanones. Zwischen beiden idealtypisch zu verstehenden Kanonformen sind, so Kärjä, fließende Grenzen zu beobachten, die permanent ausgehandelt werden. Kärjäs (ebd.: 15ff.) Vorschlag, die politische bzw. musikwirtschaftliche Einflussnahme als eigenständigen, d.h. als vorgeschriebenen Kanon, zu definieren, halte ich nicht für plausibel. Erstens ist ein Mainstream-Kanon der Fans unabhängig vom wirtschaftlichen Gefüge von Massenmedien und Plattenlabels nicht denkbar. Eine Trennung erscheint daher künstlich. Zweitens wird begrifflich eine einseitige Ursache-WirkungsRelation nahegelegt. Drittens wird die Rolle des Musikjournalismus unterschätzt. Am Beispiel Eminem wird sich zeigen, dass die Beeinflussung eines Kanons durch Musiker, Musikwirtschaft und Musikkritik bzw. Musikkonsumenten komplexer ist, als es die Metapher des vorgeschriebenen Kanons suggeriert. Kärjä ist jedoch insofern zuzustimmen, als die Frage nach den institutionellen Machtverhältnissen, die insbesondere auf die Mainstream-Kanonbildung einwirken, zentral ist. Sie erhält daher in der folgenden Analyse besonderes Gewicht.

Mit Blick auf die Analyse von Kanonbildung im HipHop möchte ich schließlich zwei Aspekte hervorheben. Zum einen besteht die Gefahr, die diskursiven Inhalte einer Kanondebatte mit ihrer sozial-ökonomischen Gestalt und Funktion zu verwechseln. Wertungsdiskurse können durchaus Produkte des Mainstreams im Namen der Authentizität ablehnen und gleichzeitig zur sozial-ökonomischen Ausbildung eines Mainstream-Kanons beitragen. Genau dieses Paradoxon findet sich auch im HipHop. Denn zum Selbstverständnis der ehemaligen Subkultur gehört es, wie wir sehen werden, zwischen HipHop als System und Mainstream-Pop als feindlicher Umwelt zu unterscheiden, obwohl HipHop sozial-ökonomisch bereits längst einen eigenen Mainstream hervorbringt.

Zum anderen ist es ratsam, nicht die - aus seiner Ursprungserzählung überlieferte - Selbstbeschreibung des Genres als Gegenentwurf zum System Popmusik (Klein/Friedrich 2003: 56f.) auf die Kanonanalyse zu übertragen und im HipHop verallgemeinernd von alternativen Kanones zu sprechen. 
Vielmehr hat HipHop im Zeichen des Mainstreamings gleichermaßen genrespezifische Mainstream-Kanones wie auch, durch seine Subszenen und Subgenres, alternative Kanones ausgebildet. Um Mainstream-Kanonformation wird es im Folgenden vornehmlich gehen.

\section{Sichtbarmachung im Mainstream}

An Eminem lässt sich zeigen, wie ein Rapper zunächst im alternativen HipHop-Kanon - hier: des Detroiter Undergrounds - verweilte und dann durch musikwirtschaftliche Selektion für den Mainstream-Kanon des HipHop sichtbar und verhandelbar wurde. Es war der Rap-Produzent Dr. Dre, der Eminem berühmt machte, indem er ihn um 1998 bei seiner Plattenfirma Interscope-Aftermath (zu Universal gehörend) unter Vertrag nahm (Bozza 2005: 40f.). Es ist ein Tauschgeschäft, das für die US-HipHop-Industrie, rastlos angetrieben durch zahlreiche Rivalitäten ihrer Musiker, seit den 1990er Jahren typisch war: ${ }^{1}$ Der Produzent von Klassikern wie Straight Outta Compton (1988) und The Chronic (1992) gründete sein eigenes camp mit vielversprechenden Rappern wie Xzibit und Eminem, deren Erfolge Dres Aufenthalt im Rap-Olymp - in der Rolle als Meistermacher - verlängern sollten. Und im Tausch dafür ließ Dre seine sLegionäre an seinem Kanonstatus teilhaben. Eminem wurde so als möglicher Kanonanwärter weithin sichtbar und als Weißer im vornehmlich schwarzen HipHop-Kontext aufgewertet - sinnfällig inszeniert durch seine Aufführung des The Chronic-Klassikers »Nuthin But A G-Thang « mit Dr. Dre und Snoop Dogg bei den MTV-Awards im Jahre 1999 (Bozza 2005: 85). Weitere Multiplikatoreffekte - wie Musikpreise und Charterfolge - wurden so wahrscheinlicher.

\section{Realness, style, skills und fame als Richtschnur}

Eine solchermaßen wirtschaftlich günstige Ausgangsposition war für Eminems Aufnahme in den HipHop-Mainstream-Kanon gegen Ende der 1990er Jahre begünstigend, wäre aber allein kaum hinreichend gewesen, wie andere Beispiele belegen. ${ }^{2}$ Neben der institutionellen Sichtbarmachung war es

1 Es scheint hier einen Zusammenhang zwischen der Battle-Struktur des HipHop und der sich immer weiter ausdifferenzierenden Independent-Major-Struktur (mehrheitlich von HipHoppern geführte HipHop-Labels mit Major-Anbindung) zu geben.

2 Man denke etwa an den Rapper AZ. 
für ihn wohl gleichermaßen entscheidend, sich für den Wertungsdiskurs, der Kanones produziert, aussichtsreich zu positionieren. Das scheint Eminem zwischen 1998 und 2002, in den Jahren seines steilen Aufstiegs, äußerst erfolgreich gelungen zu sein. Die besondere Herausforderung für ihn als Musiker in der Arena des HipHop-Mainstream bestand allerdings darin, dass der Wertekosmos des HipHop sich in dieser Zeit in einer Umbruchphase befand und von sehr unterschiedlichen Wertvorstellungen geprägt war.

HipHop wurde damals von vielen Fans und aktiven Rappern immer noch als szenebezogene Existenzweise und nicht als Modetrend betrachtet, weshalb Rap in HipHop-Magazinen nach wie vor primär an seiner Glaubwürdigkeit, Qualität und Originalität gemessen wurde - sicher mit Blick auf die Fans und ihr Bedürfnis nach Identifikation und Orientierung. Kembrew McLeod kann in seiner diskursanalytischen Untersuchung der US-HipHopSzene aus dem Jahre 1999 sogar belegen, dass sich das Wertungskriterium realness um 1998 großer Popularität erfreute, kurioserweise zu einem Zeitpunkt, an dem sich die ehemalige musikwirtschaftliche Randerscheinung HipHop mit Subkulturstatus in einen ertragreichen Geschäftszweig des USPop-Mainstreams verwandelte (McLeod 1999: 136). Die Zahlen der Recording Industry Association of America können McLeods Befund bestätigen: Bereits im Jahr 1999 schloss Rap mit über zehn Prozent Anteil am US-Markt mit Genres wie Country oder RnB auf und belegte Platz zwei in den Verkaufsstatistiken hinter Rock mit circa 25 Prozent Marktanteil (RIAA 2003). McLeod deutet die - quer zur empirischen Wirklichkeit stehende - Konjunktur der realness im HipHop als den typischen Versuch einer Musikkultur, die sich der Gefahr der Mainstream-Assimilation ausgesetzt sieht, ihre Authentizität im besonderen Maße diskursiv zu untermauern. Werte des alternativen Kanons sollten für den HipHop-Mainstream-Kanon erhalten werden, um eine Abgrenzung zur feindlichen Umwelt des so genannten Pop-Mainstreams zu gewährleisten. ${ }^{3}$ HipHop tat dies in verschiedenen Themenfeldern wie gender oder race, in denen scharf zwischen real und fake differenziert wurde. McLeod übersieht jedoch, sicher bedingt durch fehlende historische Distanz, dass das Mainstreaming nicht spurlos am HipHop-Kanon vorübergegangen war. Bereits Ende der 1990er Jahre wurde nämlich längst ein anderes Kriterium für Fans und Kritiker zunehmend wichtig und akzeptiert: fame und zwar in Gestalt kommerziellen Erfolges. Wurde zu diesem Zeitpunkt noch vorausgesetzt, dass eine gewisse Balance mit den anderen genannten Kriterien gewahrt wurde (George 2006: 262ff.), ging im neuen Jahrtausend die-

3 Assmann (2005: 123) weist auf diesen allgemeinen Zusammenhang von Komplexitätssteigerung der Umwelt, Angst vor Identitätsverlust und Kanonisierungsprozessen hin. 
ses fragile Gleichgewicht weitgehend verloren, mit der Folge, dass heute der materialistische Aspekt den US-HipHop-Diskurs allmählich bis tief in die Fanbasis hinein zu dominieren scheint. ${ }^{4}$

Die Mainstream-Kanonformation im US-HipHop des ausgehenden 20. Jahrhunderts, so lautet meine These, ist also von dieser spannungsvollen Diskurskonstellation zwischen Authentizität und kommerziell legitimierter Popularität geprägt. Im Folgenden möchte ich zeigen, dass Eminems Musik und Image unmittelbar an diese Wertungsdiskurse anknüpfen. Eminem scheint sich an dem polarisierten Erwartungshorizont, am Kanon als Richtschnur, regelrecht abzuarbeiten, affirmativ wie negierend, um seine Kanonwürdigkeit zu beweisen. Wie sehr der Rapper übrigens die Aufnahme in die »Hall of Fame ersehnte, insbesondere durch The Source, zeigt diese Äußerung aus dem Jahr 2001:

»Jeder Rapper, ganz besonders ich, träumt davon, einmal im Leben in der Quotable-Spalte der Source aufzutauchen. Als die Source damals rauskam, war es auch für mich die HipHop-Bibel. Als Erstes schlugen meine Freunde und ich immer die Quotable-Seite auf, um nachzusehen, wer jeweils dabei war. Derjenige war dann unser Gott des Monats« (Eminem in Bozza 2005: 116f.).

Um nun das Verhältnis von Eminem zu den Wertungsdiskursen im HipHop näher bestimmen zu können, beziehe ich mich im Folgenden auf McLeods "semantic dimensions", die dieser als die zentralen Themen im RealnessDiskurs um 1998 herausgearbeitet hat.

\begin{tabular}{|l|c|c|}
\hline Semantic Dimensions & Real & Fake \\
\hline Social-psychological & staying true to yourself & following mass trends \\
Racial & Black & White \\
Political-economic & the underground & commercial \\
Gender-sexual & hard & soft \\
Social-locational & the street & the suburbs \\
Cultural & the old school & the mainstream \\
\hline
\end{tabular}

Abb. 1: »HipHop-Realness« (McLeod 1999: 139)

Betrachtet man die Text-, Klang- und Bildsprache Eminems vor diesem Hintergrund, so fällt auf, in welch hohem Maße der Rapper die vom Diskurs geforderten Anforderungen an realness, aber auch an skills und style erfüllt:

4 Man denke etwa an den Fan-Diskurs anlässlich des Wettkampfes zwischen Kanye West und 50 Cent um die meist verkauften Alben im Herbst 2007. Fans identifizieren sich anscheinend zunehmend mit der Rolle des privaten Musikmanagers (Maclnnes 2007). 
So bedient Eminem in vielen seiner Songs etwa das Klischee des brutalen und sexistischen Rappers. Sein Alter Ego Slim Shady stellt der HipHopCommunity seine uneingeschränkte Bereitschaft zu hasserfüllter Härte unter Beweis, kombiniert mit sarkastischem, Pulp-Fiction-haftem, absurdem Humor. In den Songs "Kim« (2000) oder "97' Bonnie And Clyde« (1999) schreckt er z.B. nicht vor der detailliert beschriebenen Ermordung seiner Ex-Frau zurück.

Eminems zweites, autobiographisch inszeniertes lyrisches Ich, Marshall Mathers, thematisiert hingegen, was Slim Shadys bis zum Wahnsinn gesteigerten Hass motiviert: »l'm tired of being white trash, broke and always poor« (»If I Had«, 1999). Durch die Dialektik beider Rollen, Slim und Marshall, kann Eminem gleichermaßen glaubwürdig wie originell erscheinen. Die scheinbar im realen Leben wurzelnde Wut kennzeichnet Slims originelles Storytelling in »Guilty Conscience oder »Kim« als tragische Maskerade, die in ihrer biographischen Notwendigkeit an Eindringlichkeit und Glaubwürdigkeit gewinnt. Und die aus Marshalls Perspektive komponierten, anerkannten Erzählstücke wie »Cleanin Out My Closet« (2002) dürften durch den Effekt der Demaskierung noch authentischer wirken und beweisen, dass Eminem sich letztlich - in der Diktion McLeods - »treu bleibt «.

Die Kunstfiguren Slim und Marshall helfen dem weißen Rapper Eminem aber vor allem angesichts einer nahezu unlösbaren ethnischen Problematik. Denn realness ist im US-HipHop-Diskurs weitgehend für Afroamerikaner reserviert, auch wenn die Realität der amerikanischen HipHop-Community Ende der 1990er Jahre längst von diesem Mythos abweicht (Kitwana 2005: 70). Eminem nimmt die zu erwartende Kritik seiner Gegner vorweg und parodiert sich als weißer Schulversager und ungeschickter Batman-SupermanAntiheld gleich selbst (»Without Me«, 2002). Es ist der selbstzerstörerische Blick auf seine Hautfarbe, die die schwarzen Kritiker von The Source, lange bevor es zum Eklat zwischen beiden Parteien kommt, beeindruckt: Es ist der Selbsthass einer verlorenen Jugend, den auch die schwarze Erzählung von HipHop kennt (Parker 2002). Problematisch bleibt in diesem Zusammenhang dennoch Eminems fehlende street credibility, da ihm der zentrale Mythos, das schwarze Ghetto (Klein/Friedrich 2003: 53-57), als lokale und soziale Referenz nicht zur Verfügung steht.

Weiteres symbolisches Kapital kann er jedoch als ehemaliger BattleUnderground-Rapper akkumulieren. Welchen Status Eminem hier innehatte, zeigt, dass »Without Me« von The Source im Jahre 2002 aufgrund seiner treffsicheren Punchlines als herausragender Battle-Rap bewertet wurde (Osorio 2002: 170). Folgerichtig scheint, dass Eminem ausgerechnet in jenem Moment, in dem er endgültig zur zentralen Figur des als nicht-authentisch 
geltenden Pop-Mainstreams avancierte, dieses Kapital belieh: Im Kinofilm 8 Mile (US-Kinostart: November 2002) inszenierte er seine authentische Underground-Vergangenheit und die Erzählung, dass er durch seine BattleSkills die Rassengrenze überschritten habe.

In einigen Aspekten scheint Eminem sich jedoch ganz bewusst vom HipHop-Kanon abzuwenden: In der Bildsprache seiner Videos greift er vorwiegend auf das Bildarchiv weißen Pop-Mainstreams zurück (MTV-Serie South Park, Superman, Batman usw.), genau wie er in seinen Diss-Tracks neben wenig authentischen Rappern wie Will Smith oder Puff Daddy (McLeod 1999: 141ff.) vornehmlich die Popszene von Justin Timberlake über Christina Aguilera bis Britney Spears angreift, um sich möglicherweise dort, in einem zweiten Kanon, als Mainstream-konsumierbarer Rebell zu profilieren. Für diese These spricht auch die Veröffentlichungspolitik, die gezielt auf bereinigte Versionen problematischer Songs setzte bzw. Hardcore-Tracks wie »Kim« auf den Alben ersetzte (Doggett 2005: 34). Ähnliches gilt auch für die zunehmend von inm selbst produzierte Soundästhetik, in der sich ein bisweilen dominanter Rockeinfluss bemerkbar macht, insbesondere in seinen erfolgreichsten Veröffentlichungen wie The Eminem Show. Ob intendiert oder nicht, in jedem Falle sichert die Crossover-Konzeption, die gleich für zwei Kanones kompatibel ist, hohe Verkaufszahlen. Wie bedeutsam diese kommerzielle Spielart der Popularität von Anfang an für die Eminem-Rezeption ist, belegen die frühen Rezensionen in The Source, die nicht nur Eminems musikalischen, im Underground geschulten Fähigkeiten ihren Respekt bekunden, sondern zugleich seinen imposanten Millionenverkäufen (PoulsonBryant 1999: 175ff.; The Source 2006: 64ff.). Ohne jenen kommerziellen Erfolg, so meine Vermutung, wäre Eminem für den HipHop-Mainstream-Kanon am Übergang zum 21. Jahrhundert nicht verhandelbar gewesen.

Nur hatten die Verkaufsstatistiken, wie Eminem in seinem Song »White America« (2002) freilich selbst einräumt, den Makel, als Ergebnis weißen Networkings und weißer Rezeption zu erscheinen. Mit Blick auf die HipHopBeats ist es erstaunlich, dass Eminem der schwarzen HipHop-Community so wenig Kompensation für seine Etablierung im, aus HipHop-Sicht, falschen Kanon anbietet. Nur ganz selten finden sich Songs mit einem weniger glatten Sounddesign, die geeignet wären, nicht nur inhaltlich, sondern auch klanglich urbane HipHop-Kultur zu repräsentieren. So etwa das von Dr. Dre und Mel-Man produzierte »Remember Me« (2000), das den Klang von Graffiti-Sprühdosen und vorbeifahrenden Zügen einfängt und vor allem im Chorus die Stimmen übersteuert. »Remember Me« ist jener Song Eminems, der dem Genre des »Reality Rap« (Krims 2001: 70-75) klanglich am nächsten kommt. Es scheint so, als appelliere Eminem mit der Forderung »Remember 
Me« unmittelbar an das kollektive Gedächtnis der HipHop-Gemeinschaft. Die Geräusche einer Sprühdose sowie intertextuelle Bezüge ${ }^{5}$ sind nicht nur Referenzen für Insider und kennzeichnen Eminem selbst als einen solchen, sondern sie sind auch Metaphern der szenebezogenen Erinnerungspraxis: Graffiti als urbane Geschichtsschreibung. In »Till I Collapse (2002) setzt Eminem diese Technik der Selbsteinschreibung in den Kanon fort. Hier formuliert er - mit HipHop-typischem boasting - seine eigene Musikerliste, in der er sich neben Ikonen des Rap, wie Tupac, B.I.G. oder Nas, einreiht. ${ }^{6} \mathrm{Er}$ tut dies auf reimtechnisch und rhythmisch so hohem Niveau, als wolle er dies musikalisch, in Form einer Bewerbung für den Kanon, rechtfertigen. $\mathrm{Zu}$ fragen ist nun, auf welche Resonanz Eminems Bemühungen gestoßen sind. Ich möchte dies im Folgenden am Beispiel der wichtigsten HipHop-Zeitschrift der USA, The Source, beantworten.

\section{Kanonformation zwischen Musikkritik und Musikwirtschaft}

Das 1988 gegründete amerikanische Magazin The Source war jahrelang eine Instanz des US-HipHop, die für sich die Rolle in Anspruch nahm, Klassiker des Mainstream-Rap-Kanons zu küren, ${ }^{7}$ einen Korpus wichtiger Songtexte auszuwählen (Kolumne »Hip-Hop. Quotable«) und als glaubwürdiger Chronist der HipHop-Geschichte zu fungieren. Als Vermittler zwischen Mainstream und Underground (z.B. die Kolumnen »Unsigned Hype« oder »Independents' Day. The Celebration of Hip-Hop's National Underground Scene ) stellt sich die Zeitschrift bis heute als Sprachrohr der (vor allem afroamerikanischen) HipHop-Community dar, mit einer selbst bezifferten Auflage von knapp 500.000 Exemplaren und geschätzten neun Millionen Lesern im Jahre 2002 (The Source, H. 167 [Aug.] 2003: 45). ${ }^{8}$

Die Resonanz der Zeitschrift auf den weißen Rapper Eminem war sehr wechselhaft. In groben Zügen lässt sich die Rezeption in drei Phasen eintei-

5 Alle drei Rapper zitieren sich im Refrain selbst: RBX bezieht sich auf sein Featuring für Dr. Dres The Chronic (»Lady Of Rage«) bzw. Sticky Fingaz auf seine Beteiligung auf dem Onyx-Album Bacdafucup (beide LPs 1992); Eminem hingegen zitiert Liedzeilen aus Songs der Slim Shady LP von 1999: "Just Don't Give A Fuck« und "Still Don't Give A Fuck« bzw. aus dem Underground-Song »Take The Whole World With Me« (Bizarre feat. Pace Won \& Eminem, o.J.).

6 Zum Zusammenhang von boasting und Selbstautorisierung vgl. Klein/Friedrich 2003: 39, $169 f$.

7 »Classic « ist die höchste Bewertungskategorie des Record Report.

8 Tricia Rose weist bereits 1994 daraufhin, dass sich angeblich bis zu fünfzehn afroamerikanische Fans eine Zeitschrift teilen (Rose 1994: 8). 
len. Die frühe Eminem-Rezeption in The Source ab 1998 war ausgesprochen positiv, bevor es Ende 2002 zu einer Diskurswende und einer mehr als drei Jahre währenden Schmäh-Kampagne gegen den Rapper kam. 2006 war schließlich das Jahr einer erneuten Wende, als Eminem in The Source rehabilitiert wurde. Betrachten wir diese Phasen im Einzelnen.

Bereits im Frühjahr 1998 wurde Eminem in der »Unsigned Hype«Kolumne als vielversprechender MC porträtiert (Morales 1998: 46). Es folgen äußerst wohlwollende Coverstorys (Morales 2000; Parker 2002) und der Abdruck zweier Featuring-Beiträge in der »Quotable«-Kolumne. ${ }^{9} 2002$ bittet die Redaktion ihn sogar um seine Expertenmeinung zum Radio-Battle zwischen Nas und Jay-Z, einem der wichtigsten Ereignisse im US-Rap des Jahres (The Source, H. 152 [Mai], 2002: 118). Den Dokumenten ist explizit abzulesen, dass Eminems inszenierte Ambivalenz zwischen Schock-Image, witzigem Battle-Rap und autobiographischer Verletzlichkeit großen Anklang findet. Die Rezension seines dritten Albums The Eminem Show im August 2002 ist der Höhepunkt dieser Entwicklung: Kim Osorio ist voll des Lobes für seine Reime, für sein Songwriting und seinen »incredible on beat flow«. Komplexität, Unterhaltung und Realitätsbezug gingen eine gelungene Mischung ein. Positiv sei Eminems offensiver Umgang mit seiner ethnischen Herkunft (Osorio 2002). Diese ethnische Akzeptanz ist im Übrigen typisch für den RaceDiskurs, der die Eminem-Rezeption in The Source zwischen 1998 und 2002 begleitete, der zwar auch verstärkt die Ängste vor weißer Rap-Aneignung thematisierte, aber die ethnische Eingrenzung dieser Kultur ablehnte (u.a. Poulson-Bryant 1999). Negativ lastet ihm die Rezensentin hingegen seine einfallslosen Rockbeats mit »weißer Zielhörerschaft« an. Ebenso kritisch klingt an, dass die äußerst hohen Verkaufszahlen seiner Platten allein noch keine Rap-lkone aus ihm machen. Das Album erhält folglich vier von fünf Punkten (»Mics«). Der Status eines »Classics« wird verwehrt und Eminems Forderung nach Kanonisierung humoristisch aufgenommen: Die Liste würde noch weitergeschrieben, er müsse sich weiter beweisen, vor allem im Battle mit ernstzunehmenden Rappern seiner Klasse (Osorio 2002).

Wenige Monate später vollzog sich die Diskurswende: Benzino, Teilhaber von The Source und schwarzer Rapper, veröffentlichte im Herbst 2002 DissTracks gegen Eminem, die von diesem mit schmähenden Songs über seinen Herausforderer und »The Sauce beantwortet wurden (Bozza 2005: 117f.). Darauf folgte eine dreijährige Kampagne der Zeitschrift, die den weißen Rapper an den Schwachpunkten seiner Image-Konstruktion angriff: Eminem, von Benzino in »Die Another Day« als »rap Hitler « besungen, besitze keine

9 "Forgot About Dre« mit Dr. Dre (The Source, H. 124 [Jan.] 2000: 188) und »Renegade« mit Jay-Z (The Source, H. 147 [Dez.] 2001: 194). 
»street credibility « und stelle sich als »culture-stealer « dar: als »Elvis des Rap«, der als Weißer den Schwarzen die Kultur wegnehme (Benzino 2003). Das im März 2003 erschienene Themenheft Hip-Hop under attack stellte Eminem - neben der soeben verschärften polizeilichen und medialen Hetzjagd - sogar als zentrales Gefährdungsmoment der HipHop-Nation dar. Im Zentrum der Ausgabe steht ein Roundtablegespräch mit Größen des HipHopKanons zur Rettung der HipHop-Kultur. In einem von Benzino konzipierten Comic am Ende des Heftes wird Eminem bezichtigt, die schwarze HipHopCommunity an der Krönung ihrer Trinität Nas, B.I.G. und Jay-Z zu hindern und Rap durch weißen Mainstream zu unterjochen. Dres Musikkonzern Interscope soll daraufhin dafür gesorgt haben, dass mehrere Labels ihre Werbung in der Zeitschrift zurückzogen (Ford 2004: 86). Während Eminem trotz seiner kommerziellen Erfolge mit The Eminem Show und 8 Mile im Jahre 2002 und 2003 in The Source kaum noch positive Beachtung fand, betonte die Zeitschrift im Umfeld der Source Awards 2003 ihre Autorität für die Community und beschwor die Leser, nun gemeinsam HipHop-Geschichte zu schreiben (Osorio 2003) - gemeint ist freilich: ohne Eminem. Schließlich präsentierte The Source im Herbst 2003 eine alte Aufnahme, in der Eminem sich despektierlich über eine schwarze Ex-Freundin geäußert hatte (Coates 2003). Dies führte 2004 zu der von Eminem gerichtlich erfolglos angefochtenen Veröffentlichung der Aufnahme und zu einem über zwanzigseitigen Schwerpunkt im Februarheft 2004, der Eminems realness aufgrund seines weißen Rassismus zu demontieren suchte und bereits bekannte Motive fortspann: Eminem stehe für die Monopolisierung der HipHop-Wirtschaft, da sein Mutterkonzern Interscope, dem mittlerweile auch die von Eminem entdeckte Schlüsselfigur des Jahres 2003, 50 Cent, angehörte, nicht nur den Plattenmarkt beherrsche, sondern auch das Musikfernsehen MTV, ${ }^{10}$ die Radios und die Vertriebswege. The Source sei demnach das letzte Bollwerk der schwarzen Indie-Label gegen die weiße Mainstream-Industrie (Osorio 2004; Anon. 2004a).

Wie ist diese Rezeptionswende zu erklären? Bakari Kitwana, bis April 1999 The Source-Redakteur und schwarzer Bürgerrechtler, nennt in seinem

10 The Source behauptete, dass MTV durch die 1999 neu eingeführte Video Music Award-Kategorie »HipHop « weiße Musiker protegieren wolle - zu Ungunsten schwarzer Rapper, denen seither die Kategorie »Rap« zugeteilt würde (Anon. 2004a: 73). Die Liste der Gewinner der Kategorie »HipHop« von 1999 bis 2006 zeigt keine Dominanz von Musikern nicht-afroamerikanischer Herkunft: Black Eyed Peas mit »My Humps « (2006), Missy Elliott feat. Ciara \& Fat Man Scoop mit "Lose Control« (2005), OutKast mit »Hey Ya!« (2004), Missy Elliott mit »Work It « (2003), Jennifer Lopez feat. Ja Rule mit »I'm Real« (2002), OutKast mit »Ms. Jackson« (2001), Sisqó mit »Thong Song« (2000), Beastie Boys mit »Intergalactic « (1999) (MTV 2007). 
2005 erschienenen Buch Why White Kids Love Hip-Hop psychologische und soziologische Gründe. Zum einen seien Benzino und David Mays als Inhaber der Zeitschrift von Neid getrieben: Benzino aufgrund seiner musikalischen Erfolglosigkeit, trotz jahrelang verborgener Teilhaberschaft an The Source, Mays aufgrund seiner Angst, die Position als weiße Schüsselfigur im schwarzen Rap-Kontext an einen anderen Weißen zu verlieren. Interessant ist, dass Benzino dies teilweise sogar eingesteht (Ratcliff 2004: 84). Zum anderen habe das Phänomen Eminem zu einer »collision between America's old and new racial politics « geführt (Kitwana 2005: 136). Zwar habe es gegen Ende des Jahrtausends viele jüngere Schwarze gegeben, die die Integration weiBer HipHop-Musiker befürworteten (ebd.: 137), ${ }^{11}$ nicht zuletzt befördert durch die soziale Angleichung des weißen Prekariats an die Lebenswirklichkeit schwarzer Jugendlicher (ebd.: 23ff., 28, 31-34). Im Fall Eminems habe aber bei den Kritikern das Denken der älteren Generation, ihre Erfahrung mit der unversöhnten US-amerikanischen Geschichte und ihre Furcht vor weißer Vorherrschaft und kultureller Ausbeutung die Überhand gewonnen (ebd.: 137). Ich stimme Kitwana in seiner Analyse grundsätzlich zu. Und natürlich war es provokant, dass Eminem 2002 zur dominanten Figur des (weißen) Pop-Mainstreams avancierte, weil sein Hardcore-Image in The Eminem Show und vor allem in dem Film 8 Mile aufgeweicht und konsumierbar gemacht wurde. Nach anfänglicher Ablehnung avancierte Eminem plötzlich zur Ikone der amerikanischen Öffentlichkeit (Bozza 2005: 150-161, 352). Er führte mit mehr als 7,6 Millionen verkauften Alben gegen den Trend der CD-Marktkrise den Pop-Mainstream an (RIAA 2003) ${ }^{12}$ und hatte gleichzeitig, wie Chuck D Ende 2002 diagnostizierte, neben Nas und Jay-Z keine ernst zu nehmende lebende Konkurrenz im Mainstream des HipHop (Chuck D in Bozza 2005: 257f.). Dass Eminem ab 2003 seine Position durch die Allianzen mit 50 Cent und The Game weiter ausbaute, verschärfte diese Situation. ${ }^{13}$ Es erscheint nicht unwahrscheinlich, dass Osorios fast trotzig wirkende Kritik am kommerziellen Erfolg von The Eminem Show - ein zu diesem Zeitpunkt veraltetes Argument - auch durch die überwältigende Mediendominanz Eminems verstärkt wurde. Dass The Source aber ab Ende 2002 die »old racial politics« gegen Eminem förmlich ins Feld führte, hatte

11 Die Berichterstattung von The Source vor 2002 zeigte, wie gesehen, viele solcher Ansätze, möglicherweise auch als Erbe Kitwanas Tätigkeit als politischer Redakteur.

12 US-Umsatzrückgang im Bereich CD-Alben: 2000-2001: -6,4\%; 2001-2002: -8,9\%.

1350 Cents Album Get Rich Or Die Tryin' (2003) verkaufte sich mehr als sechs Millionen Mal und war das erfolgreichste Pop-Album des Jahres. The Massacre (2005) verkaufte sich fünf Millionen Mal (The Source 6/2006: 67, 69), The Documentary (2005) von The Game bis Ende 2005 2,3 Millionen Mal (Anon. 2006). 
noch einen anderen Grund: Das Magazin befand sich anscheinend genau zu dem Zeitpunkt, an dem die Fehde begann, in einer wirtschaftlich schwierigen Situation, die Benzino und Mays bereits Ende 2002 durch neue Investoren zu meistern suchten. Der Musikfernsehkonzern Black Entertainment Television (BET) wurde 2002 durch seine Investitionen in Höhe von zwölf Millionen US-Dollar zum stillen Teilhaber (Kelly 2006) und übernahm knapp vier Jahre später als Teil einer Investorengruppe das mittlerweile hoch verschuldete Magazin (Zambito 2006; Kelly 2006).

Ein entscheidender Grund für diese Turbulenzen war wahrscheinlich der Konkurrenzdruck durch das 1997 gegründete HipHop-Magazin XXL, das spätestens im Frühjahr 2003 zur ernsthaften Konkurrenz für The Source wurde (Arango 2003). So erklärt sich, warum The Source in einer Posterbeilage im Februarheft 2003 nicht nur Eminem angriff, sondern auf der Rückseite auch $X X L$ : Der XXL-Herausgeber Elliot Wilson, ehemaliger The Source-Redakteur, wird hier von einem schwarzen, ein The Source-Shirt tragenden Riesen malträtiert. Die Bildunterschrift lautet: »Respect The Architect«.

In diesem Lichte betrachtet kann die Eminem-Fehde als Versuch einer Zeitschrift gedeutet werden, im Wettstreit mit einem konkurrierenden Magazin verlorenen Rückhalt in der Leserschaft zurückzugewinnen. Während $X X L$ nach anfänglich reservierter Haltung gegenüber Eminem ${ }^{14}$ in den Märzheften 2003 und 2004 mit positiven Coverstorys über Dr. Dre, Eminem und 50 Cent aufmachte, setzte The Source auf Kritik an Eminem und zunehmend auch an 50 Cent. Die Hoffnung, auf diese Weise symbolisches und materielles Kapital zurückzugewinnen, erfüllte sich allerdings nicht, zu unpopulär war es wahrscheinlich, sich gegen derart erfolgreiche Rapper und ihre wirtschaftlichen Netzwerke zu stellen. Verschlimmert wurde die wirtschaftliche Lage der Zeitschrift durch eine ruinöse Personalführung und eine verschwenderische Ausgabenpolitik der Herausgeber. Nicht bezahlte Rechnungen und gerichtliche Klagen ehemaliger Mitarbeiter und Mitarbeiterinnen trugen maßgeblich zum finanziellen Kollaps der Zeitschrift bei (Kelly 2004, 2005, 2006; Zambito 2006). Zudem dürfte die Bekanntgabe von Benzinos stiller Teilhaberschaft an The Source im Januar 2002 deren Glaubwürdigkeit beschädigt haben (Crook 2002; Arango 2003), denn nun wurde publik, dass der Rapper die »Rap-Bibel« möglicherweise zum eigenen Vorteil missbraucht hatte.

Nach einem Leserverlust von $50 \%$ binnen zweier Jahre (Kelly 2005) mussten Mays und Benzino das zahlungsunfähige Magazin 2006 verlassen

14 Auf Eminems Song »Marshall Mathers« (2000; »Double XL, Double XL / Now your magazine shouldn't have so much trouble to sell«) reagierte die Redaktion mit einer kritischen Coverstory (Anon. 2000). 
(Kelly 2006). Kurz nach ihrem Weggang wurde in The Source die Geschichte des HipHop prompt neu bewertet. Eminem nahm in der Gesamtschau der besten MCs von 1988 bis 2005 eine zentrale Stellung ein. Wie kein anderer Rapper wurde er nun zweimal auf Platz eins (2000 und 2002) bzw. einmal auf Platz zwei (1999) der Jahreswertungen gelistet (The Source 2006, 63ff.).

\section{Fazit}

Der Historiker Jan Assmann spricht im Kontext von Religionsgemeinschaften und ihren Textkanones davon, dass demjenigen die Führung über den Kanon gebührt, »der die umfassendste Kenntnis und die einleuchtendste Deutung « hat (Assmann 1999: 27). Genau diese Rolle reklamierte The Source als »Bibel des HipHop« für sich. Mit Assmann könnte man zu Recht erklären, dass The Source den Kampf um Glaubhaftigkeit und Sachkenntnis gegen den von ihr einst geachteten Rapper verloren hat und ihre Führungsrolle deshalb abgeben musste. Die Analyse der Fehde zeigt jedoch, dass MainstreamKanonformation im HipHop auch durch wirtschaftliche Machtstrukturen mitbestimmt wird: Beide Akteure müssen zwar in der Öffentlichkeit ihre Kompetenz und Glaubwürdigkeit je in ihrem Medium unter Beweis stellen. Doch zusätzlich gilt es für beide, den Einfluss auf den Kanon machtstrategisch zu sichern. Während sich die Erfolgschancen für The Source durch journalistische Konkurrenz, falsche Allianzen und Misswirtschaft strukturell verschlechterten, stärkte Eminem seine Position, indem er, wie einst sein Mentor, ein eigenes Wirtschaftsimperium aufbaute. Seine Selbstpositionierung zwischen realness, skills, style und fame bzw. seine Vernetzung mit Ikonen der Vergangenheit sowie mit Stars der Gegenwart machte es für die geschwächte Institution The Source nahezu unmöglich, ihn im Zeitraum von 2002 bis 2006 aus dem Kanon zu streichen.

\section{Literatur}

Anon. (2000). »Message To A White Man.«In: XXL, H. 5 (August), S. 95-100.

Anon. (2004a). "The Making of a Hip-Hop Monopoly. «In: The Source, H. 173 (Februar), S. 73.

Anon. (2004b). "Clap Back: Representatives of the Hip-Hop Nation Sound Off On Eminem and Racism. «In: The Source, H. 173 (Februar), S. 80f.

Anon. (2006). "The Year's Top Albums in Sales « In: The New York Times vom 6. Januar. Online unter: http://www.nytimes.com/2006/01/06/arts/music/ 06bnich.html?adxnnl=1\&adxnnlx=1136802104-/Kl38/Bi5hWM/3CeuLOfbg

(Zugriff: 19.1.2008). 
Appen, Ralf von / Doehring, André (2006). »Nevermind The Beatles, Here's Exile 61 and Nico: 'The Top 100 Records of All Time - A Canon of Pop and Rock Albums From a Sociological and an Aesthetic Perspective." In: Popular Music 25, H. 1, S. 21-39.

Arango, Tim (2003). »Rap Bible Bashed. The Source Hit Hard on Newsstand by Rival XXL. «In: The New York Post vom 19. Mai, S. 35.

Assmann, Jan (1999). Fünf Stufen auf dem Wege zum Kanon. Tradition und Schriftkultur im frühen Judentum und seiner Umwelt. Münster: Lit.

Assmann, Jan (2005). Das kulturelle Gedächtnis. Schrift, Erinnerung und politische Identität in frühen Hochkulturen. München: Beck (5. Aufl.; 1. Aufl. 1992).

Benzino (2003). »Die Another Day. «In: The Source, H. 161 (Februar), S. 132.

Bozza, Anthony (2005). Whatever you say I am. Eminem. Die Biographie. München: Heyne (US-amerik. Original 2003).

Coates, Ta-Nehisi (2003). »Caught On Tape.« In: The Village Voice vom 21. November. Online unter: http://www.villagevoice.com/music/0348, coates2, 48918,22.html (Zugriff: 9.12.2007).

Crook, Dara (2002). »Benzino. The Benzino Project.« In: The Source, H. 148 (Januar), S. 148.

Doggett, Peter (2005). Eminem. The Complete Guide To His Music. New York: Omnibus Press.

Ford, Ryan (2004). »The Realist. «In: The Source, H. 182 (November), S. 84-88.

George, Nelson (2006). XXX. Drei Jahrzehnte HipHop. Freiburg: orange press (erw., aktual. und ill. Neuausgabe; US-amerik. Original 1998).

Kärjä, Antti-Ville (2006). »A Prescribed Alternative Mainstream: Popular Music and Canon Formation. « In: Popular Music 25, H. 1, S. 3-19.

Kelly, Keith J. (2004). »Vendors Go to the Source over Claims of Unpaid Bills. «In: The New York Post vom 14. April, S. 32.

Kelly, Keith J. (2005). "The Source of Confusion. «In: The New York Post vom 28. Oktober, S. 44.

Kelly, Keith J. (2006). "Hip-Hop Heave-Ho - New Board Ousts Top Dogs At The Source. « In: The New York Post vom 19. Januar, S. 36.

Kitwana, Bakari (2005). Why White Kids Love Hip-Hop. Wangstas, Wiggers, Wannabes, and the New Reality of Races in America. New York: Basic Civitas Books.

Klein, Gabriele / Friedrich, Malte (2003). Is this real? Die Kultur des HipHop. Frankfurt/M.: Suhrkamp.

Krims, Adam (2001). Rap Music and the Poetics of Identity (= New Perspectives in Music History and Criticism). Cambridge: Cambridge University Press (Nachdruck der 1. Aufl. 2000).

MacInnes, Paul (2007). »Bling for Your Supper: Hip-Hop Stars Go Into Battle Over the Future of a Stuttering Genre. «In: The Guardian vom 1. September. Online unter: http://www.guardian.co.uk/uk_news/story/0,,2160224,00.html (Zugriff: 9.12.2007).

McLeod, Kembrew (1999). »Authenticity Within Hip-Hop and Other Cultures Threatened with Assimilation. «In: Journal of Communication 49, H. 4, S. 134-150.

Morales, Riggs (1998). »Unsigned Hype. Eminem.« In: The Source, H. 102 (März), S. 46.

Morales, Riggs (2000). »American Psycho.«In: The Source, H. 130 (Juli), S. 180-187. MTV (2007). [Video Music Award-Gewinner]. Online unter: http://www.mtv.com/ ontv/vma/archive/winners.jhtml (Zugriff: 9.12.2007). 
Osorio, Kim (2002). »Eminem. The Eminem Show.«In: The Source, H. 155 (August), S. $169 f$.

Osorio, Kim (2003). »History in The Making.«In: The Source. Special issue: Official Guide to the Source Awards, S. 12.

Osorio, Kim (2004). »The Real Slim Shady.« In: The Source, H. 173 (Februar), S. 70-78.

Parker, Erik (2002). »The Last Laugh.«In: The Source, H. 152 (Mai), S. 112-118.

Poulson-Bryant, Scott (1999). "Fear of a White Rapper."In: The Source, H. 117 (Juni), S. 174-177, 180, 186.

Ratcliff, Fahiym (2004). "Last Line of Defense. [Interview mit Benzino]. In: The Source, H. 173 (Februar), S. 82-85, 97f.

RIAA (2003). The Recording Industry Association of America's 2003 Yearend Statistics. Online unter: http://www.riaa.org/keystatistics.php (Zugriff: 9.12.2007).

Rose, Tricia (1994). Black Noise. Rap Music and Black Culture in Contemporary America. Middletown: Wesleyan University Press.

Smith, Pam (2005). »Behind Closed Doors at The Source Magazine. "Online unter: SOHH.com, 22. März, www.sohh.com/article_print.php?content_ID=6861 (Zugriff: 19.1.2008).

The Source (2006). Special Collectors Edition, H. 200 (Juni).

Zambito, Thomas (2006). "Judge Lops Hip-Hop Mag Bias Award. «In: New York Daily News vom 1. November. Online unter: http://www.nydailynews.com/archives/ news/2006/11/01/2006-11-01_judge_lops_hip-hop_mag_bias_.html (Zugriff: 19. 1.2008).

\title{
Diskographie
}

Dr. Dre (1992). The Chronic. Death Row Records, LC 11645, SPV 085-94022.

Eminem (1999). The Real Slim Shady LP. Aftermath Ent./Interscope Records, 490 287-2.

Eminem (2000). The Marshall Mathers LP. Aftermath Ent./Interscope Records, 490 629-2.

Eminem (2002). The Eminem Show. Aftermath Records, 493290-2.

N.W.A. (1988). Straight Outta Compton. Priority Records, 72435-37936-2-3.

\begin{abstract}
The article presents an analysis of the sbeef between Eminem and the hip-hop magazine The Source between 2002 and 2006. The potential impact of Eminem's rap music on the hip-hop canon and his image are examined. The role of The Source as one of the most important institutions of forming this canon is discussed. This study shows that both, aesthetical and socio-economical aspects play an important role for canon formation as far as mainstream hip-hop in the USA is concerned.
\end{abstract}

